\title{
A REVIEW OF ONLINE CONSUMER BEHAVIOUR RESEARCH: MAIN THEMES AND INSIGHTS
}

\author{
Inma Rodríguez-Ardura, University of Oxford, United Kingdom, and Internet Interdisciplinary Institute, Open University of \\ Catalonia, Spain \\ Francisco J. Martínez-López, University of Granada and Open University of Catalonia, Spain \\ Juan C. Gázquez-Abad, University of Almería, Spain \\ Gisela Ammetller, Internet Interdisciplinary Institute, Open University of Catalonia, Spain
}

\begin{abstract}
We offer a structured vision of the main research contributions made on a wide set of issues compounding the online consumer behaviour area. We present a wide view of research topics, analyzed from the marketing perspective, from the early nineties till now. This approach may be useful, not only to better structure or reinforce the knowledge academics have accumulated in the field, but also to serve as a guide and source of ideas for future investigation.

We base on three broad purposes underlying the online consumer's studies to group and present the literature review: profiling of Internet users and shoppers; issues related with the online consumer behaviour -including the determinants of the decision to buy online, the online satisfaction and loyalty, and the adoption of classic theories and models to explain Internetbased consumer behaviour; and, more recently, issues related with the consumers' empowerment and the co-creation of value.

Within this last wave of research, mostly connected with the emergence of social networking applications, we situate a collection of studies examining the forms in which the new digital tools empower the consumer; the consumer participation in collective initiatives of innovation and generation of content; the consumer behaviour in personalization processes; and the determinants and consequences of consumer participation in (brand) virtual communities. The discussions we present allow seeing how the emerging area of the online consumer behaviour is enriching the marketing disciplines as well as facilitating the advance of customer-centric paradigms. In addition, we provide strategic insights about marketing programmes on the Internet.

Finally, several insights and research opportunities are highlighted and discussed, for instance: 1) exploring sources of online empowerment, which may increase the consumers' capabilities to participate in setting and developing the firms' value propositions; 2) assesing the effectiveness of the strategies used to benefit consumers from a collective intelligence and to give them greater control over their shopping experiences; 3 ) analyzing the critical aspects of current practices to provide website personalization to consumers, as well as the possibilities offered when 'personalising the personalisation'; and 4) exploring the effects of participating in brand communities (and, also, anti-brand communities) on brand-related variables (e.g. trust and loyalty).

References available upon request.
\end{abstract}

